\title{
Us and them
}

\author{
Cindy A. Buckmaster, PhD, CMAR, RLATG
}

I recently read that the formation of identity is based on an interplay between two basic, human needs: our need to belong and our need to be unique. By this characterization, we want to be like others and be one of a kind at the same time. This got me thinking about causes and the zealotry that often springs from groups that form around them. As a high school teacher years ago, I marveled at the intense drive of teenagers to distinguish themselves from their peers, to carve out their own beliefs and philosophies about life. Once they sorted out their positions on things, they worked the crowds, pulling others who were struggling with this same need for clarity and distinction into ideological discussions about what is right and what is wrong in the world. At some point cultures emerged from collectives, complete with rules to live by and leaders who demanded fierce loyalty. Group identities were born that allowed these young people to be unique and to belong at the same time, a brilliant solution for fulfilling these basic, human needs. Group members felt connected and proud. They were confident in their pleas and attestations, a confidence supported by safety in numbers. Those who defended their group's doctrine became 'us'; those who did not became 'them'. When complex issues arose, causes emerged to address them and debates ensued about who was right and who was wrong. Passion, anger, blame and hatred fortified the identities of opposing groups, and the remaining stragglers felt compelled to choose a side to believe and defend.

This dynamic has shaped human behavior throughout history. We see it in sports. We see it in religion. We see it in politics. We have a strong desire to

align with one side or another whenever we navigate complex issues in life. That's healthy. That's human. But when our chosen side becomes our identity, that's zealotry. And when this happens, we lose our souls. Nothing we think or feel is pure or loving anymore. Our view of reality is mangled by fear and fanaticism and the only thing we truly love... is hate. As biomedical research professionals we've endured the hatred of animal rights fanatics for years. These people thrive on anger and anarchy. They aren't interested in facts. They prefer fantasies. They have lost their humanity. They are doctrines. And their survival depends on forcing their ideology on the rest of us, regardless of the pain and suffering this brings to animals and people.

\section{While both sides shove their agendas down our throats to protect their group identities, our animals remain voiceless, depending on the rest of us to protect their welfare.}

A few months ago, I read an article in the New York Times that described atrocious claims of animal neglect and mistreatment at a federally funded agricultural research center. According to this report, thousands of animals died of starvation and hundreds died from painful, treatable infections. One of the most grotesque allegations involved a young cow with her head locked in place to keep her immobile while six bulls mounted her for hours. Her body was "torn up" and her back legs were broken. She remained in that condition for hours, until she died. The investigation was extensive. Hundreds of open records documents were reviewed and several research professionals were interviewed about their experiences in this center. The lack of oversight and animal suffering described was unbearable for me to consider.

Moments after reading this article, I saw a thread about it on social media by a group that strongly opposes animal rights fanatics for all of the reasons noted above. These were some of their comments: "What a bunch of BS... It sounds exactly like what it is-someone's trying to make something out of nothing... They're just trying to paint the industry in a bad light... I smell a rat... If the writer had legit concerns, you can't tell it from the usual two-bit animal rights wolfcrying." These people dismissed everything they read as lies, reflexively. They justified some of the horrors described, and they implied that others were fabrications. They never considered, for a moment, that any of the animal suffering described could be real. There wasn't a trace of concern in any of their words, and it was clear that the only thing they truly loved... was hate. They have lost their humanity. They have become a 'side', a mirror image of the zealotry they oppose, and neither side is interested in what really happened in this research center. While both sides shove their agendas down our throats to protect their group identities, our animals remain voiceless, depending on the rest of us to protect their welfare. Extremism in every form is an unforgivable diversion from our ethical obligations. Our animals need us to keep our promise to care for them with compassion and respect. They need us to live in truth. And the truth has no sides. 